\title{
ESTUDIO PSICOMÉTRICO dE LA ESCALA DE COSTO Conductual de Compras ECológicas en MÉxico*
}

\author{
JoRge PALACIOS-DELGADO \\ http://orcid.org/0000-0002-4351-5667 \\ Universidad del Valle de México, Querétaro, México \\ Red de Investigación del Comportamiento del Consumidor \\ CRISTINA VANEgAS \\ http://orcid.org/0000-0002-2266-986X \\ Universidad Nacional Autónoma de México, FESZ, Ciudad de México, México \\ Red de Investigación del Comportamiento del Consumidor \\ JosÉ BUSTOS \\ http://orcid.org/0000-0003-3423-596X \\ Universidad Nacional Autónoma de México, FESZ, Ciudad de México, México \\ Red de Investigación del Comportamiento del Consumidor
}

Correo electrónico: drjpalacios81@gmail.com

Recibido: 21 de septiembre del 2021 / Aceptado: 21 de octubre del 2021

doi: https://doi.org/10.26439/persona2021.n024(2).5444

\begin{abstract}
RESUMEN. El objetivo de la presente investigación es obtener evidencias de validez basadas en la estructura interna y en relación con un criterio, así como la confiabilidad de las puntuaciones en la Escala de Costo Conductual de Compras Ecológicas, empleando una muestra de 221 personas, entre 17 y 62 años $(M=27,57 ; D E=10,8)$, de diferentes ciudades de México. Los resultados psicométricos muestran que la Escala de Costo Conductual de Compras Responsables con el Medioambiente tiene una estructura unidimensional, con un ajuste óptimo de los datos al modelo a través del AFC y una consistencia interna aceptable (alfa $=.75$; omega $=.75$ ). Además, el costo conductual permite diferenciar a quienes realizan bajas acciones proambientales de quienes realizan acciones a favor del medioambiente. La discusión analiza la utilidad de los resultados en la evaluación del costo conductual dentro del comportamiento responsable con

\footnotetext{
* Declaramos que no existe conflicto de interés por parte de los autores del estudio. El financiamiento del artículo se deriva de la colaboración con la Red de Investigación del Comportamiento del Consumidor, integrada por varias universidades públicas y privadas de México. Finalmente, agradecemos a los participantes del Seminario de Psicología de la Universidad del Valle de México, campus Querétaro, por su colaboración en la distribución y aplicación de los instrumentos de medición.
} 
el ambiente, así como las implicaciones para futuras investigaciones sobre psicología ambiental y económica.

Palabras clave: psicología económica / compras ambientalmente responsables / costo personal / escala y conducta proambiental

\title{
PSYCHOMETRIC ANALYSIS OF THE BEHAVIORAL COST SCALE OF GREEN PURCHASE IN MEXICO
}

\begin{abstract}
The purpose of this study was to obtain validity evidence based on the internal structure and based on a criterion and reliability estimated of the scores derived from the Green Purchasing Behavioral Cost Scale in a sample of 221 participants between 17 and 62 years old $(M=25,57 ; S D=10,8)$ in México. Data analysis revealed an unidimensional structure that characterizes green purchasing behavioral cost and acceptable internal consistency (alfa $=.75$; omega $=.75$ ); confirmatory factor analysis showed optimal fit of the data to the model. Additionally the behavioral cost scores distinguish between individuals with low pro-environmental actions from those who perform actions in favor of the environment. The discussion analyzes the utility of the results for assessment of personal cost within responsible behavior with the environment, as well as the implications for future research into the pro-environmental and economic psychology.
\end{abstract}

Keywords: economic psychology / consumer green purchase / scale and pro-environmental behavior 


\section{INTRODUCCIÓN}

Entre las múltiples opciones que las personas tienen para ayudar a conservar el ambiente (Palacios \& Bustos, 2012), se encuentran las compras verdes o ecológicas, que se refieren a la adquisición de productos considerando de forma responsable el impacto que generan sobre el ambiente y eligiendo aquellos que lo afectan en menor medida (Joshi \& Rahman, 2015). Al igual que otras acciones incluidas en el conjunto de conductas proambientales (CPA), la realización de compras ecológicas puede verse impedida por la evaluación del costo que tiene para la persona (Pieters, 1987; Steg \& Abrahamse, 2013), entendido este como requerimientos y pérdidas que tendrá el individuo si decide llevar a cabo la conducta, en este caso, la adquisición de un producto ecológico (Pieters, 1991; Vanegas \& Bustos, 2019). Si bien se ha observado que los individuos tienden a realizar una conducta proambiental si esta no representa costos importantes para ellos en términos de dinero, tiempo, esfuerzo y conveniencia (Diekmann \& Preisendörfer, 2003), las conductas proambientales varían en su exigencia y demanda para llevarlas a cabo. Aunque el costo más evidente podría ser el económico, es decir que la opción verde sea de mayor precio (Joshi \& Rahman, 2015; Rezvani et al., 2015), otros elementos que resaltan son la calidad o la sustituibilidad, que se refiere a que el producto funcione igual que las opciones tradicionales no ecológicas (Gupta \& Ogden, 2009). En el caso de compras verdes, es importante saber cómo se comportan estas en función de variables socioeconómicas, psicosociales, conductuales y también contextuales (Blankenberg \& Alhusen, 2019).

La evaluación del costo de la CPA se presenta de diferentes formas en la literatura: el uso de expertos para jerarquizar acciones (Andersson \& Von Borgstede, 2010), el empleo de la frecuencia de realización (Diekmann \& Preisendörfer, 2003; Kaiser \& Schultz, 2009) o la generación de reactivos sobre desventajas específicas para la conducta por evaluar (Black et al., 1985; Pieters, 1987). Vanegas (2018) desarrolló dos escalas del costo de la CPA (como requerimientos y como consecuencias) con la intención de que sean aplicables a diferentes acciones favorables para el ambiente; en particular, la escala de costos como consecuencias ha sido retomada en otros estudios con resultados variados, puesto que no se observó relación significativa con el consumo de agua (Manríquez et al., 2016) y las compras verdes (Vanegas \& Bustos, 2019); pero sí se apreció un efecto de mediación/ moderación entre conducta sustentable y percepción de consecuencias positivas (Corral et al., 2020), así como una relación negativa con la ejecución de conductas proambientales variadas (Vanegas, 2018).

Por otra parte, las compras verdes también forman parte del estudio del comportamiento del consumidor (Griskevicius et al., 2010; Young et al., 2010). En México, se puede apreciar cada vez más investigaciones que buscan profundizar en su conceptualización a partir de, por ejemplo, la caracterización de las compras en términos de los aspectos 
definitorios del consumo sustentable (Barrientos, 2014), su significado (Soler et al., 2017), los rasgos de personalidad expresivos (Palacios et al., 2016) o los estados afectivos involucrados en el comportamiento de compra (Viloria \& Palacios, 2019). Mientras que en la evaluación se observa el desarrollo de escalas de medición que destacan las compras compulsivas (Rueda et al., 2019), la toma de decisiones impulsiva y razonada (Soler et al., 2019), las compras ambientalmente responsables (Bustos et al., 2012) y las barreras para la compra de productos ecológicos (Barrientos \& Bustos, 2019), así como un mayor interés por perfilar a los consumidores verdes (Carrete et al., 2013).

A pesar de contar con estos instrumentos de medida desarrollados y validados en el contexto mexicano, se carece de un instrumento que evalúe los costos personales del consumo ecológico; por eso, se plantea el desarrollo de una escala para este fin, considerando el comportamiento de compra de los mexicanos (Palacios \& Soler, 2017) y bajo una propuesta apoyada en la economía conductual (Kahneman, 2003; Palacios \& Bustos, 2019) que provea un marco de referencia para comprender las decisiones que tienen las personas respecto a su comportamiento económico y el tipo de compras que realizan, entre las que se encuentran las compras ecológicas.

El instrumento facilitará el estudio del costo personal y su asociación con las compras y el consumo ecológico en nuestro país. Puede convertirse en un instrumento aplicable en la investigación en psicología económica, ambiental o social, y para el seguimiento de estrategias de intervención en estos ámbitos. Por ejemplo, los economistas en el campo de los estudios empíricos se pueden beneficiar de la existencia de instrumentos válidos y confiables sobre las compras verdes (Blankenberg \& Alhusen, 2019). Además, al incorporar este constructo como parte de la economía conductual, su incidencia podrá ser parte de los modelos de cambio de conducta; por lo anterior, el objetivo de la investigación fue obtener evidencias de validez basada en la estructura interna y evidencias de validez basada en la relación con otra variable (criterio), así como obtener los niveles de confiabilidad de la Escala de Costo Conductual de Compras Ecológicas.

\section{MÉTODO}

\section{Diseño}

Para la presente investigación se realizó un estudio de campo con un diseño de investigación no experimental de corte transversal, instrumental y psicométrico (Montero \& León, 2002).

\section{Participantes}

Se seleccionó una muestra no probabilística de manera intencional de 221 personas, $58,8 \%$ mujeres y $41,2 \%$ hombres, con un rango de edad entre 17 y 62 años, con una media 
de 27,57 ( $D E=10,86)$. El 45,7 \% procede de la Ciudad de México, el 47,5\% del Estado de México y el 6,8 \% de diferentes ciudades del país (Guanajuato, Puebla, Nuevo León).

\section{Instrumentos}

\section{Escala de Costo Conductual de Compras Ecológicas}

Se adaptó la escala de costos como consecuencias de realizar conducta proambiental (Vanegas, 2018; Vanegas \& Bustos, 2019) con un lenguaje específico a las compras ecológicas (por ejemplo: "Con frecuencia son más caros que los productos tradicionales") y adecuado a la sociocultura mexicana (Palacios, 2021; Palacios \& Martínez, 2017). La adaptación tiene cinco alternativas de respuesta que van desde "totalmente de acuerdo" a "totalmente en desacuerdo". La escala original cuenta con evidencias de validez basada en la estructura interna a través del análisis factorial confirmatorio y evidencias de validez a partir de la relación con el comportamiento proambiental, así como una confiabilidad por consistencia interna (alfa de Cronbach $=.77$ ) para el puntaje total del instrumento.

\section{Escala de Conducta Proambiental}

Se utilizó la Escala de Conducta Proecológica de Corral et al. (2009). Es una escala unidimensional de trece afirmaciones con un formato de respuesta tipo Likert, en la que los participantes reportan la frecuencia de comportamientos de cuidado del ambiente con cuatro opciones de respuesta que van desde "nunca" hasta "siempre". La confiabilidad por consistencia interna alcanza un coeficiente alfa de .82 . Se cuenta además con evidencias convergentes de validez a partir de la relación con la orientación hacia la sostenibilidad.

\section{Procedimiento}

El levantamiento de datos se realizó de enero a marzo del 2020. Para la recolección de datos, los instrumentos empleados se incorporaron a un formulario digital de Google Forms. Con ayuda de investigadores de dos instituciones educativas, así como de alumnos, se compartió el enlace vía redes sociales invitando a responder de manera voluntaria, en un tiempo aproximado de diez minutos. En el formulario se incluyó el consentimiento informado y preguntas sociodemográficas sobre sexo, edad y ciudad de origen, además de los instrumentos de evaluación. Se explicó a los participantes que la evaluación tenía como objetivo conocer algunas características relacionadas con las compras y se les pidió que contestaran de forma sincera, ya que sus respuestas se utilizarían para fines de investigación. 


\section{Consideraciones éticas}

Se utilizó el consentimiento informado de los participantes. El protocolo de investigación fue establecido de acuerdo con el Reglamento de la Ley General de Salud, en su apartado sobre investigación en seres humanos (Secretaría de Salud, 2011). Asimismo, el protocolo de trabajo está registrado ante el Comité de Investigación de la Universidad del Valle de México, campus Querétaro, con el código de registro PCSUVM0112018.

\section{Análisis de datos}

Se realizaron análisis descriptivos de los ítems de la Escala de Costo Conductual de Compras Ecológicas (media, desviación estándar, asimetría y curtosis). Para examinar las evidencias de validez basada en la estructura interna, se puso a prueba un modelo de medición con ecuaciones estructurales estableciendo indicadores de ajuste absoluto ( $\left.X^{2}, R M S E A\right)$, de ajuste incremental (CFI, TLI, RFI, IFI) y de ajuste de parsimonia (NFI), de acuerdo con los criterios establecidos por Hu y Bentler (1999). Para determinar el ajuste del modelo, se siguieron las directrices publicadas por la European Journal of Psychological Assessment (Schweizer, 2010). Adicionalmente, se calculó la confiabilidad por consistencia interna a través de los coeficientes alfa de Cronbach $(\alpha)$ y omega de McDonald ( $\omega)$ (Viladrich et al., 2017).

A continuación, se analizaron descriptivamente las características de la distribución de las variables estudiadas (media, desviación estándar, rango). En el caso de la Escala de Conducta Proambiental, el análisis descriptivo se hizo para la sumatoria total de los reactivos. Se examinó la bondad de ajuste a la distribución normal con la prueba de Shapiro-Wilk. Posteriormente, para obtener evidencias de validez referidas a un criterio, se realizaron comparaciones con la prueba $U$ de Mann-Whitney para analizar las diferencias de la escala entre quienes tienen un alto y bajo comportamiento proambiental (CPA). Los análisis de datos se efectuaron con los programas SPSS y AMOS (Arbuckle \& Wothke, 1999).

\section{RESULTADOS}

En la tabla 1 se presentan los resultados de los análisis descriptivos de los ítems de la Escala de Costo Conductual de Compras Ecológicas.

Para poner a prueba la estructura del instrumento original, se contrastó el modelo de medida unidimensional por medio de un análisis factorial confirmatorio aplicando el método de estimación de la máxima verosimilitud. Se consideraron los índices de modificación para encontrar el ajuste, de modo que el modelo original de 10 ítems podía derivar en un modelo de 8 ítems eliminando los ítems 1 y 4 . A continuación, se procedió a calcular el modelo solo con los 8 ítems. 
Tabla 1

Estadísticos descriptivos de los ítems de la Escala de Costo Conductual del Consumo Ambiental

\begin{tabular}{|c|c|c|c|c|}
\hline & Media & $D E$ & Asimetría & Curtosis \\
\hline $\begin{array}{l}\text { C1. Algunos productos tienen una presentación } \\
\text { menos agradable*. }\end{array}$ & 3,23 & 1,07 & $-0,17$ & $-0,42$ \\
\hline $\begin{array}{l}\text { C2. Con frecuencia son más caros que los } \\
\text { productos tradicionales. }\end{array}$ & 4,19 & 0,93 & $-1,29$ & 1,57 \\
\hline $\begin{array}{l}\text { C3. Al usarlos no se obtiene un resultado } \\
\text { como el que acostumbro (por ejemplo, en los } \\
\text { detergentes). }\end{array}$ & 3,29 & 0,99 & $-0,20$ & $-0,40$ \\
\hline $\begin{array}{l}\text { C4. Algunos productos parecen de baja } \\
\text { calidad*. }\end{array}$ & 3,25 & 1,02 & $-0,12$ & $-0,74$ \\
\hline $\begin{array}{l}\text { C5. Son difíciles de conseguir en las tiendas o } \\
\text { el supermercado. }\end{array}$ & 3,90 & 0,97 & $-0,84$ & 0,28 \\
\hline $\begin{array}{l}\text { C6. Me cuesta trabajo identificar aquellos que } \\
\text { son cuidadosos con el ambiente. }\end{array}$ & 3,57 & 1,17 & $-0,44$ & $-0,86$ \\
\hline $\begin{array}{l}\text { C7. Gasto demasiado tiempo buscando la } \\
\text { opción ecológica. }\end{array}$ & 3,30 & 1,07 & $-0,30$ & $-0,53$ \\
\hline C8. Implica un esfuerzo adicional conseguirlos. & 3,69 & 1,00 & $-0,70$ & $-0,02$ \\
\hline $\begin{array}{l}\text { C9. Existen pocos productos ecológicos que yo } \\
\text { pueda comprar. }\end{array}$ & 3,55 & 1,01 & $-0,45$ & $-0,46$ \\
\hline $\begin{array}{l}\text { C10. El envase, empaque o etiqueta son poco } \\
\text { llamativos. }\end{array}$ & 3,17 & 1,04 & $-0,10$ & $-0,51$ \\
\hline
\end{tabular}

Nota. Los ítems marcados con * fueron eliminados de la versión final por medio de los índices de modificación.

Elaboración propia

En la tabla 2, se muestran los resultados de ambos modelos. Se observa que el modelo estimado de 8 ítems mostró un ajuste óptimo a los datos. Los indicadores de bondad de ajuste absoluto, de ajuste incremental y de ajuste de parsimonia mostraron ser adecuados y superiores al modelo de 10 ítems (Hu \& Bentler, 1999; Pérez et al., 2000). El factor latente proporciona una adecuada explicación de los indicadores observables, con coeficientes lambda que van de .37 hasta .67 (véase la figura 1). Los análisis de consistencia interna mostraron un coeficiente $\alpha=.75$ (IC95 \% $=.71-.78$ ) y un coeficiente $\omega=.75$ (IC95 \% = .71-.79) considerados como aceptables. 
Tabla 2

Indicadores de ajuste de los modelos de medida

\begin{tabular}{ccccccccc}
\hline Modelo & $X^{2}$ & $X^{2} / d f$ & CFI & TLI & NFI & RFI & IFI & RMSEA \\
\hline Modelo con 10 ítems & 116.40 & 4.2 & .89 & .85 & .86 & .82 & .89 & 0.09 \\
Modelo con 8 ítems & 57.75 & 2.5 & .93 & .90 & .89 & .85 & .93 & 0.06 \\
\hline
\end{tabular}

Elaboración propia

Figura 1

Análisis factorial confirmatorio de la Escala de Costo Conductual de Compras Ecológicas

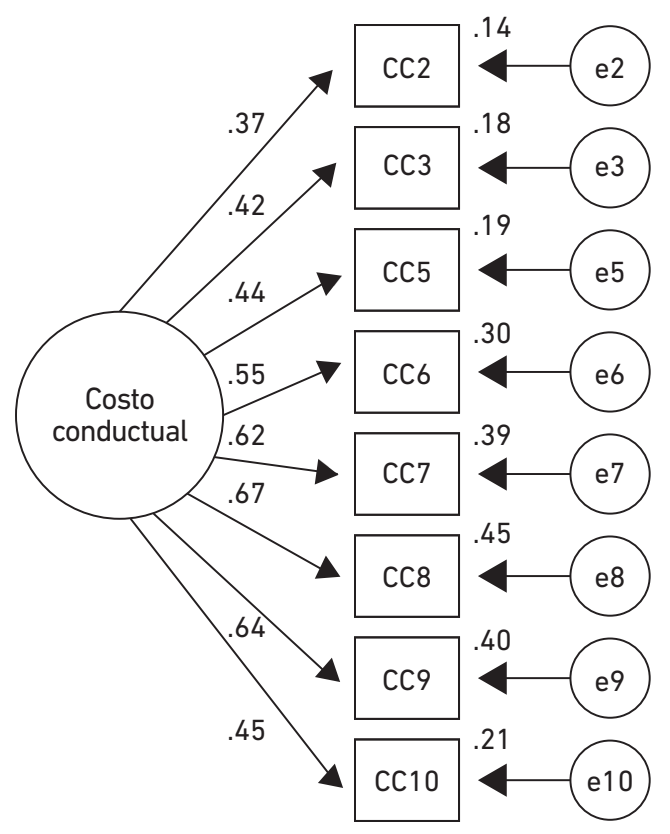

Elaboración propia

El análisis descriptivo de la Escala de Costo Conductual indica que en la muestra analizada se obtuvo una media de $27,07(D E=5,2)$, una asimetría de $-0,12$ y una curtosis de $-0,14$, con una puntuación mínima de 13 y máxima de 40 . Se consideraron los cuartiles para identificar puntos de referencia de niveles del costo conductual que tienen los participantes, lo que dio como resultado un bajo costo conductual (puntuación de 13 a 23), medio bajo (puntuación de 24 a 27), medio alto (puntuación de 28 a 30) y alto (puntuación de 31 a 40) costo conductual. Las puntuaciones presentaron una distribución que no se aproxima a nivel poblacional a la distribución normal (S-W $[g l=365]=.99, p=.044$ ) 
Se realizó el análisis descriptivo de la CPA en los participantes. La escala tiene una media de 34,01 ( $D E=6,4)$ y una mediana de 34,00. Cuenta con una asimetría de .027 y una curtosis de -.117. Una puntuación mínima de 13,00 y una máxima de 50,00. Se consideró la puntuación de la mediana para dividir a la muestra en los que tuvieran un bajo nivel de CPA (puntuación de 13 a 34) y un alto nivel de CPA (puntuación de 35 a 50), lo que dio como resultado que el 46,8 \% tuviera una valoración baja de CPA y un 53,2 \% alcanzara una valoración alta de CPA.

Se comparó los puntajes obtenidos en la Escala de Costo Conductual en función de los niveles de conducta proambiental (alta o baja) a través del contraste $U$ de Mann-Whitney. Los resultados mostraron diferencias estadísticamente significativas $(U=13$ 810,50; $Z=-2,76, p=.006, r=.19)$, observándose que la puntuación total es mayor para los que no son proambientales $(M d=28,00, M=28,46, D E=4,7, R P=199,24)$ en comparación con los que sí realizan un comportamiento proambiental $(M d=27,00, M=26,46, D E=5,3$, $R P=168,69)$.

\section{DISCUSIÓN}

La investigación en conducta proambiental suele centrarse en factores que promueven una acción de interés. En menor medida, se analizan los factores que la limitan o impiden, como el costo conductual (Pieters, 1987). El costo conductual ha sido señalado como un factor motivacional del comportamiento proambiental (Steg \& Vlek, 2009) que ayuda a entender la discrepancia entre la disposición y la acción efectiva (Blankenberg \& Alhusen, 2019; Diekmann \& Preisendörfer, 2003); no obstante, recibe menos atención que otras variables como las normas o los valores ambientales. Respecto a la medición del constructo de costo conductual, parece haber una carencia de instrumentos psicométricos para evaluar el constructo, utilizando solamente algunos reactivos u otros criterios (Andersson \& Von Borgstede, 2010; Diekmann \& Preisendörfer, 2003). En el presente estudio, se ha tomado como punto de partida una escala que evalúa el costo de realizar una CPA en general (Vanegas, 2018; Vanegas \& Bustos, 2019), para plantear la propuesta de una versión específica para las compras verdes.

Para estimar el grado en que el instrumento adaptado mide el costo conductual de compras ecológicas, se evaluaron dos aspectos de su validez. En primer lugar, los resultados obtenidos con el modelo estimado proporcionan evidencias de validez basadas en la estructura interna de la medida del costo conductual de compras ecológicas a través de los indicadores que lo integran. Los ítems fueron elaborados para medir este constructo a partir del instrumento original (Vanegas \& Bustos, 2019) desde una perspectiva etnopsicológica (Palacios, 2021; Palacios \& Martínez, 2017). En este estudio se corrobora la estructura unidimensional consistente con la versión que sirvió de base (Vanegas, 2018; Vanegas \& Bustos, 2019). Su confirmación estructural se suma al conjunto de 
instrumentos desarrollados en México (Bustos et al., 2012; Rueda et al., 2019; Soler et al., 2019; Vanegas, 2018) que permiten identificar factores antecedentes de las compras ecológicas.

Además, los pesos factoriales significativos y elevados en la relación indicadorvariable latente reafirman que el costo conductual puede ser medido por una serie de ítems agrupados en un factor que le otorga congruencia conceptual. Al examinar el contenido de los ítems que integran la escala, podemos observar ítems que refieren al tiempo, esfuerzo o trabajo adicional que se tiene que realizar para poder identificar o conseguir los productos amigables con el ambiente, además de que el precio es más elevado que el de los productos convencionales o contaminantes. Por otra parte, hallamos afirmaciones que describen mala calidad, mal funcionamiento o un empaque menos atractivo que los productos que adquieren las personas. En suma, el contenido de los ítems refleja distintos comportamientos que limitan la compra de productos ecológicos contra los contaminantes.

En cuanto al análisis de consistencia interna, los coeficientes alfa de Cronbach y omega de McDonald alcanzados indican un nivel aceptable de confiabilidad. Sin embargo, se deberán revisar las afirmaciones con la finalidad de mejorar su contenido, aumentar su confiabilidad e incorporar posiblemente una mayor variedad de comportamientos que incorporen la cantidad de esfuerzo y tiempo para poder identificar y adquirir productos ecológicos en las compras. Será necesario aumentar los niveles de confiabilidad, sobre todo si se pretende vincular la escala obtenida con variables de la psicología económica, o bien con variables ambientales.

Por otra parte, considerando la media de cada afirmación, podemos observar aquellas que se encuentran por encima de la media teórica y que pueden mostrar cuáles son representativas del constructo. Así, por ejemplo, vemos que las afirmaciones que tienen la media más alta son las siguientes: "Con frecuencia son más caros que los productos tradicionales", lo que concuerda con otros estudios que citan en primer lugar la parte económica (Joshi \& Rahman, 2015; Rezvani et al., 2015); en segundo lugar, se tiene: "Son difíciles de conseguir en las tiendas o el supermercado"; y, en tercer lugar, se ubica la aseveración: "Implica un esfuerzo adicional conseguirlos", lo que apoya la noción de que el costo personal implica, además del precio, un esfuerzo extra para encontrar los productos amigables con el ambiente (Barrientos \& Bustos, 2017; Blankenberg \& Alhusen, 2019; Bustos et al., 2012; Carrete et al., 2013; Joshi \& Rahman, 2015). Estudios correlacionales con instrumentos mexicanos como las decisiones de compra impulsiva y razonada (Soler et al., 2019), las compras ambientalmente responsables (Bustos et al., 2012) o con preferencias de compra (Palacios \& Bustos, 2019) complementarán la noción sobre requerimientos o pérdidas que tendrán que llevar a cabo si deciden adquirir un producto ecológico.

Para obtener evidencias de validez referida a un criterio de la medición — propuesta en este estudio-, se utilizó como criterio la CPA como lo plantean otros estudios (Bustos 
et al., 2012; Corral et al., 2009; Palacios \& Bustos, 2012). Los datos encontrados sugieren que el costo conductual de compras ecológicas es capaz de diferenciar entre quienes realizan CPA y quienes no, observándose que aquellos que no realizan acciones de conservación ambiental puntúan más alto en los costos percibidos, como han encontrado otros estudios (Corral et al., 2020; Pieters, 1987; Vanegas \& Bustos, 2019). Este resultado es acorde con la idea de que la consideración de costos impide la ejecución de conductas (Steg \& Abrahamse, 2013), lo cual aporta evidencia de validez de criterio.

Por otro lado, de la información obtenida, se derivan implicaciones prácticas, debido a que la escala evaluada puede ser útil para hacer frente al cambio climático, aportando información a propuestas y políticas de consumo de productos verdes dirigidas a mitigar el impacto de la producción de bienes y servicios tradicionales (no verdes) en el ambiente. Uno de los modos que parece idóneo para abordar esta problemática es mediante intervenciones realizadas para tal fin. Tales estrategias de educación ambiental deberán contemplar aspectos como los planteados en este estudio, si se pretende obtener efectos positivos en dichas intervenciones.

Es importante señalar una serie de limitaciones del estudio. Una de ellas reside en el instrumento, particularmente en los niveles de confiabilidad, por lo que en próximos estudios será necesario analizar los ítems a fin de mejorar la consistencia interna del instrumento, con la finalidad de obtener resultados más certeros y fiables. Otra limitación tiene que ver con el tamaño de la muestra, que para futuros estudios tendrá que ampliarse; asimismo, es necesario replicar el estudio en diversas muestras con la finalidad de corroborar la estructura teórica del instrumento adaptado para este estudio, su fiabilidad y su vinculación con la conducta económica o ambiental.

Para finalizar, podemos señalar que la aportación de este estudio fue obtener evidencias de validez basadas en la estructura interna y a partir de las relaciones con otras variables, así como estimar la consistencia interna de la evaluación del costo personal por medio de una variable latente de manera válida, confiable y culturalmente sensible para medir este constructo en una muestra de habitantes de México. Ajzen (1991) señala que un problema fundamental en la baja capacidad de predicción de las variables antecedentes está en el nivel de especificidad con que normalmente se miden, en comparación a la CPA que se quiere predecir. Por tanto, la generación de una Escala de Costo Conductual enfocada en la compra de productos ecológicos es una herramienta más precisa para el estudio de esta conducta proambiental. Antes de concluir, nos gustaría mencionar que futuros estudios nos permitirán conocer el efecto que tiene el costo conductual sobre las compras ecológicas; en este sentido, nuestra próxima línea de investigación será probar modelos que incorporen este constructo dentro de las decisiones ambientales y económicas. 


\section{REFERENCIAS}

Ajzen, I. (1991). The Theory of Planned Behavior. Organizational Behavior and Human Decision Processes, 50, 179-211.

Andersson, M., \& Von Borgstede, C. (2010). Differentiation of Determinants of Low-Cost and High-Cost Recycling. Journal of Environmental Psychology, 30(4), 402-408. https://doi.org/10.1016/j.jenvp.2010.02.003

Arbuckle, J. L., \& Wothke, W. (1999). Amos 4.0 User's Guide. SPSS.

Barrientos, C. (2014). Facilidades y barreras para el consumo sustentable. En J. M. Bustos \& L. M. Flores (Eds.), Psicología ambiental, análisis de barreras y facilidades psicosociales para la sustentabilidad (pp. 137-156). PAPIIT UNAM. https://www. researchgate.net/publication/328116754_Psicologia_ambiental_analisis_de_ barreras_y_facilidades_psicosociales_para_la_sustentabilidad

Barrientos, C., \& Bustos, J. M. (2017). Predictores psicológicos de la compra de productos ecológicos en habitantes de la Ciudad de México. Alternativas en Psicología, 38, 129-144. https://alternativas.me/27-numero-38-agosto-2017-enero-2018/161predictores-psicologicos-de-la-compra-de-productos-ecologicos-enhabitantes-de-la-ciudad-de-mexico

Barrientos, C., \& Bustos, J. M. (2019). Evaluación de barreras para la compra de productos ecológicos en habitantes de la Ciudad de México. Revista Nthe, 26, 56-64. http:// nthe.mx/NTHE_v2/pdfRevistas/PDF_Revista20200226164614.pdf

Black, J. S., Stern, P. C., \& Elworth, J. T. (1985). Personal and Contextual Influences on Household Energy Adaptations. Journal of Applied Psychology, 70(1), 3-21. https:// psycnet.apa.org/doi/10.1037/0021-9010.70.1.3

Blankenberg, A. K., \& Alhusen, H. (2019). On the Determinantsof Pro-Environmental Behavior: A Literature Review and Guide for the Empirical Economist [cege Discussion Papers, No. 350]. University of Göttingen, Center for European, Governance. Economic Development Research (cege), Göttingen. http://hdl. handle.net/10419/204821

Bustos, J. M., Palacios, J., Barrientos, C., \& Flores, L. M. (2012). Validez de la escala de consumo ambientalmente responsable. El Psicólogo Anáhuac, 15, 11-17.

Carrete, L. P., González, E. M., Centeno, E. A., Castaño, R. M., \& Felix, R. (2013). ¿Qué características tienen los consumidores verdes en México? Un enfoque sobre segmentación demográfica fundamentada en las 3R's y la compra de productos ecológicos. Estudios Gerenciales, 30(132), 287-300

Corral, V., Robles, K., Corral, N. S., Hernández, B., \& Suárez, E. J. (2020). Variables que afectan la relación entre las conductas sustentables y sus consecuencias 
psicológicas positivas: rasgos de personalidad y costos conductuales. Psicumex, 10(1), 1-20. https://doi.org/10.36793/psicumex.v10i1.336

Corral, V., Tapia, C., Frías, M., Fraijo, B., \& González, D. (2009). Orientación a la sostenibilidad como base para el comportamiento pro-social y pro-ecológico. Medio Ambiente y Comportamiento Humano, 10(3), 195-215.

Diekmann, A., \& Preisendörfer, P. (2003). The Behavioral Effects of Environmental Attitudes in Low-Cost and High-Cost Situations. Rationality and Society, 15(4), 441-472. https://doi.org/10.1177/1043463103154002

Griskevicius, V., Tybur, J. M., \& Van den Bergh, B. (2010). Going Green to Be Seen: Status, Reputation, and Conspicuous Conservation. Journal of Personality and Social Psychology, 98(3), 392-404. https://doi.org/10.1037/a0017346

Gupta, S., \& Ogden, D. T. (2009). To Buy or Not to Buy? A Social Dilemma Perspective on Green Buying. Journal of Consumer Marketing, 26(6), 376-391. https://doi. org/10.1108/07363760910988201

Hu, L.T.,\&Bentler,P.M.(1999).CutoffCriteria forFitIndexes in Covariance Structure Analysis: Conventional Criteria versus New Alternatives. Structural Equation Modeling: A Multidisciplinary Journal, 6(1), 1-55. doi.org/10.1080/10705519909540118

Joshi, Y., \& Rahman, Z. (2015). Factors Affecting Green Purchase Behaviour and Future Research Directions. International Strategic Management Review, 3(1-2), 128-143. http://dx.doi.org/10.1016/j.ism.2015.04.001

Kahneman, D. (2003). A Psychological Perspective on Economics. American Economic Review, 93, 162-168. http://doi:10.1257/000282803321946985

Kaiser, F. G., \& Schultz, P. W. (2009). The Attitude-Behavior Relationship: A Test of Three Models of the Moderating Role of Behavioral Difficulty. Journal of Applied Social Psychology, 39, 186-207. https://doi.org/10.1111/j.1559-1816.2008.00435.x

Manríquez, J. C., Corral, V., \& Vanegas, M. C. (2016). Positive (Gratitude, Eudaimonia) and Negative (Scarcity, Costs) Determinants of Water Conservation Behavior. PsyEcology, 7(2), 178-200. https://doi.org/10.1080/21711976.2016.1149986

Montero, I., \& León, O. G. (2002). Clasificación y descripción de las metodologías de investigación en psicología. Revista Internacional de Psicología Clínica y de la Salud, 2(3), 503-508. https://www.redalyc.org/pdf/337/33720308.pdf

Palacios, J. (2021). Evidencias de validez y confiabilidad de la escala de flexibilidad en jóvenes mexicanos. Persona, 24(1), 27-45. https://doi.org/10.26439/persona2021. n024(1).5311 
Palacios, J., \& Bustos, J. M. (2012). La teoría como promotor para el desarrollo de intervenciones psicoambientales. Psychosocial Intervention, 21(3), 245-257. https://doi.org/10.5093/in2012a22

Palacios, J., \& Bustos, J. M. (2019). Decisiones financieras y aversión al riesgo desde una perspectiva de la economía conductual. Revista Nthe, 26, 18-25. http://nthe.mx/ detalleArt.php?id=103

Palacios, J., Bustos, J. M., \& Soler, F. L. (2016). Personalidad en diferentes niveles del comportamiento de compra. En R. Díaz-Loving, L. I. Reyes, S. Rivera, J. E. Hernández y R. García (Eds.), Aportaciones actuales de la psicología social (vol. III, pp. 898-904). AMEPSO.

Palacios, J., \& Martínez, R. (2017). Descripción de características de personalidad y dimensiones socioculturales en jóvenes mexicanos. Revista de Psicología, 35(2), 453-484. doi.org/10.18800/psico.201702.003

Palacios, J., \& Soler, F. L. (2017). Preliminary Study of the Comparative Profile of Financial Control in University Young People. Ciencias Psicológicas, 11(2), 201-212. https:// doi.org/10.22235/cp.v11i2.1491

Pieters, R. G. M. (1987). Perceived Costs and Benefits of Buying and Using a Subsidized Compost Container. Resources and Conservation, 14, 139-154. https://doi. org/10.1016/0166-3097(87)90018-6

Pieters, R. G. M. (1991). Changing Garbage Disposal Patterns of Consumers: Motivation, Ability, and Performance. Journal of Public Policy \& Marketing, 10(2), 59-76. http:// www.jstor.org/stable/30000236

Pérez, J. A., Chacón, S., \& Moreno, R. (2000). Validez de constructo: el uso de análisis factorial exploratorio-confirmatorio para obtener evidencias de validez. Psicothema, 12(Supl. 2), 442-446. http://www.psicothema.com/psicothema. asp?id=601

Rezvani, Z., Jansson, J., \& Bodin, J. (2015). Advances in Consumer Electric Vehicle Adoption Research: A Review and Research Agenda. Transportation Research Part D: Transport and Environment, 34, 122-136, http://dx.doi.org/10.1016/j. $\operatorname{trd} .2014 .10 .010$

Rueda, D. A., Palacios, J., \& Sánchez, M. (2019). Estudio preliminar de la escala de compras compulsivas en Querétaro: análisis de validez y confiabilidad. Revista Nthe, 26, 40-48. http://nthe.mx/detalleArt.php?id=102

Schweizer, K. (2010). Some Guidelines Concerning the Modeling of Traits and Abilities in Test Construction. European Journal of Psychological Assessment, 26(1), 1-2. https://doi.org/10.1027/1015-5759/a000001 
Secretaría de Salud. (2011). Reglamento de la Ley General de Salud en Materia de Investigación para la Salud. http://www.salud.gob.mx/unidades/cdi/nom/compi/ rlgsmis.html

Soler, F. L., Bustos, J. M., Palacios, J., Zeelenberg, M., \& Díaz-Loving, R. (2019). Development and Validation of the Inventory of Emotional and Reasoned Purchases Decision-Making Styles (PDMI). Suma Psicológica, 26(2), 75-85. https://doi. org/10.14349/sumapsi.2019.v26.n2.3

Steg, L., \& Abrahamse, W. (2013). How to Promote Energy Savings among Households: Theoretical and Practical Approaches. En V. Corral, C. H. García y M. Frías (Eds.), Psychological Approaches to Sustainability (pp. 61-80). Nova Science Publishers.

Steg, L., \& Vlek, C. (2009). Encouraging Pro-Environmental Behavior: An Integrative Review and Research Agenda. Journal of Environmental Psychology, 29(3), 309-317. https://doi.org/10.1016/j.jenvp.2008.10.004

Vanegas, M. C. (2018). El papel de la percepción de costos y beneficios en la relación entre factores disposicionales y comportamiento proambiental [Tesis doctoral, Universidad Nacional Autónoma de México]. https://repositorio.unam.mx/ contenidos/61139

Vanegas, M. C., \& Bustos, M. (2019). Relación del costo personal y creencias ambientales con compras verdes. Revista Nthe, 26, 1-6. http://nthe.mx/detalleArt.php?id=99

Viladrich, C., Angulo-Brunet, A., \& Doval, E. (2017). A Journey around Alpha and Omega to Estimate Internal Consistency Reliability. Annals of Psychology, 33(3), 755-782. https://doi.org/10.6018/analesps.33.3.268401

Viloria, O. E., \& Palacios, J. (2019). Estados afectivos del consumidor en México: comparación entre hombres y mujeres. Revista Nthe, 26, 26-32. http://nthe.mx/ detalleArt.php?id=101

Young, W., Hwang, K., McDonald, S., \& Oates, C. J. (2010). Sustainable Consumption: Green Consumer Behaviour when Purchasing Products. Sustainable Development, 18(1), 20-31. https://doi.org/10.1002/sd.394 